\title{
Psycho-social aspects of medical abortion: why women do it? - A study in urban women of Uttarakhand.
}

\author{
Dr. Anjali Choudhary \\ Assistant Professor, Department of OBGYN, SGRRIM\&HS, Dehradun, Uttarakhand, India
}

\begin{abstract}
Since its introduction, medical abortion has rapidly gained popularity. The ease of administration ,the convenience, wider availability of drugs -sometimes over the counter - low complication rates, and the affordable cost has led to the of abuse medical abortion [M.A.]. Objective of the study was to see why women resort to abortion without hesitation, what is their knowledge, attitude and mindset about medical abortion. We subjected 124 women seeking medical abortion to a questionnaire comprising their educational social ,religious background, reasons for seeking abortion, contraceptive usage, acceptability of post- abortion contraception. Observations; .Most of women were married. Common cause pregnancy was non use of contraceptive, followed by failed contraception. Reasons for wanting abortion was too soon after LCB, having completed family, too early in the married life, economic conditions and wanting to postpone pregnancy for education and career. Conclusion; Medical abortions is being looked as an easy way to get rid of an unintended pregnancy. More women are taking pro-choice rather than pro-life approach. The guilt of abortion and moral liability is reducing, because of easy availability. Women opt for medical abortion instead of using regular contraception, as a means of post conception method of limiting and spacing families.
\end{abstract}

Keywords - Abuse, Alternative to contraception, medical abortion, Psycho-social aspects

\section{INTRODUCTION}

Every year 80 million women experience unintended pregnancies occur world-wide, out of which 46 million are terminated, $90 \%$ of these terminations take place in the first trimester [1]. Majority of women are resorting to termination of pregnancies by drugs because it is safe, easy, effective, avoids surgery and its attendant complications .It is convenient, - requires no admission, minimal follow up, and low level skill of service provider.

Since the approval of mifepristone and misoprostol protocols for induction of abortion in early pregnancies, almost a decade ago- in 2002- medical abortion [M.A] has rapidly become popular. Government of India has made amendments to preexisting MTP act of 1971, in 2003 to accommodate medical abortion. [1]. Within a year of introduction,M.A entered the main stream gynecology practice and by $2004,84 \%$ of Gynecologists were aware of and prescribing medical abortion drugs [2]. Four million M.A are performed every year. Ease of administration, wider accessibility of drugs - sometimes over the counter - low complication rates and the affordable cost has led to the of abuse medical abortion drugs, to the point of being used at times as an alternative to contraception. Gynecologists advocating termination of pregnancy with drugs as a safer alternative to surgical abortion also has done a lot to popularize the method [3] women are exercising 'pro-choice' rather than the 'pro-life' approach to abortion. Local pharmaceutical companies are also playing a major role in promoting M.A.

Mifepristone (also known as RU 486) was developed in France in the late 1980s. Combined with a prostaglandin it can be used for medical abortion and does reach an efficacy rate of well over $90 \%$ if used at early gestational age. Approved in France in late 1988, other European countries such as Great Britain and Sweden as well as China followed soon. In India it is approved since 2002. Today an estimated $30 \%$ of all abortions in the country are performed using this procedure. In India medical abortions drugs are widely available in pharmacies as a kit containing five tablets, one tablet of Mifepristone $200 \mathrm{mg}$, and four tablets of misoprostol 200 mcg each. Millions of such kits are sold every year making India largest consumer, only second to China in extent of use[1].

Many studies over the last decade focused on safety, efficacy, dosage and medical squeal of abortion with the mifepristone-prostaglandin-protocol, conclude that it is a safe and effective protocol for medical termination, with a wide acceptability and a better alternative to surgical abortions $[3,6]$. The present study was designed to understand why women resort to M.A., what social, psychological, economical reasons drive them to take the step instead of using a reliable contraception to prevent such a predicament- which is much easier-. What are the attitudes and the mind set of these women who consider unintended pregnancies as a nuisance that should be rid of at the earliest. 


\section{DATA COLlECTION CRITERIA}

From a sample of Medical abortion cases, we selected 124 women seeking medical abortion. Only those women eligible for the Medical abortion i.e. intrauterine pregnancy of 49 days or less were included in the study. Pre treatment Ultrasound was done to confirm gestational age and intrauterine pregnancy.

\section{METHODOLOGY}

We conducted a survey with help of a questionnaire shown in the figure 1. The data was collected over two visits. In the first visit the key areas of the data collection survey were.

1.: Obstetric History

-. Social background

*.: Religious beliefs

Education level

- Contraceptive Use

Reasons for seeking abortion

Emotional factors

Willingness to use post abortion contraception

Additionally the subject's background knowledge, prior use, attitude and acceptance of M.A. were assessed. Women were also asked about their willingness to use the M.A. again in future if similar conditions arise. All women were given oral mifepristone $200 \mathrm{mg}$ on day one followed 24 hours later by 800 micrograms of misoprostol, per vaginum or per oral. An USG was done 15 days later to check completeness of abortion. Upon completion of abortion, we asked the women about their experience, any excess pain or bleeding. Their emotional status regarding, guilt, stress anxiety and fear about future fertility was questioned and this information was included in our survey.

\section{SURVEYED DATA AND OBSERVATIONS}

1. Demographics of women -- out of 124 women 118 were married and 5 unmarried. Gravidity - Primi Gravida $-12,2^{\text {nd }}$ gravid -46 , 3rd Gravida $-50,4^{\text {th }}$ Gravida+ - 16 . Hindus-- 102 , Muslims--15, Sikhs--5, other religion 2 . We found that women voluntarily came seeking M.A. 72\%, had prior information and 26$\%$ had used the method before. All women were educated urban women with minimum education of matriculation and maximum of post graduation. When given an option between surgical and medical abortion most women opted for M.A, mainly for its convenience .Many of the women were not even using contraceptives before, only $25 \%$ reported pregnancy as failure of contraception. $90 \%$ women had no guilt or apprehension for choosing to abort.

2. Reasons for seeking abortion were, failure of contraception - 36, too soon after the last delivery - 50, completed family - 36, wanting to postpone pregnancy due to education or career needs - 6 , out of wedlock pregnancies - 5. When we tried to counsel them about prolife choices and requested continuation of pregnancies, $92 \%$ responded in favor of abortion, carrying on with an unwanted pregnancy was found to be more painful than termination.

3. Family support - About $89 \%$ women had the support of the spouse. Approximately $7 \%$ of the partners did not know about the pregnancy at all. Most women had not disclosed their pregnant status to their families. Surprisingly some of the lower middle class women were very agitated and wanted a fast resolution to their predicament as the pregnancies were the result of domestic abuse in the marital frame, saying they could not avert sexual advances by their spouses. There was a general sense of urgency and anxiety in most women, as if this pregnancy was an unwanted nuisance to be rid of as soon as possible.

4. Medical abortion - Of the 124 women opting for mifepristone, plus misoprostol protocol for abortion, $90 \%$ had a complete abortion without complications. Mean duration of bleeding was15.2 days. Majority perceived pain as tolerable, only $8 \%$ needed analgesic. $9 \%$ needed either a second dose of misoprostol or surgical procedure to complete the abortion. There was a group of cases wherein women had self administered the M.A drugs purchased from local pharmacy. Some of these women needed additional doses of misoprostol to control bleeding. In the pre-abortion counseling, $80 \%$ women agreed to adopt some form of contraception after abortion (because we did not agree to provide M.A. without their promise to use some form of reliable contraception for the future). However only few of them came for follow up and accepted family planning method. $20 \%$ were reluctant to use contraception, saying that 'they will manage ', or that 'they don't need it.' Some said 'Let me abort this pregnancy, it won't happen in future'. Some women were even willing to undergo M.A again without any qualms.

5. Psycho-social status - The degree of guilt and a sense of moral obligation to life, that we expected women would have towards their pregnancies, was sadly missing. Only a few women were guilty, and anxious. Women's attitude toward M.A. seemed too casual and popping in abortion pill was nothing more than 
taking vitamin pills. There was minimal guilt about not taking enough contraceptive precautions, willingness to use precaution in future was not among many of them .There was a sad and callous disregard towards life.

\section{DISCUSSION}

Medical abortion offers an easy way put an end to a life that was unintended, conveniently. Combining mifepristone with misoprostol achieves a abortion rate of almost $95 \%$. In our study there was $88 \%$ rate of complete abortion \& $12 \%$ needed surgical evacuation or a second dose of misoprostol. Ulmann, A., Silvestre, L., Chemama et al report a large-scale trial with mifepristone (RU 486) followed by the administration of a prostaglandin with a overall success rate $95.3 \%$ irrespective of dose and route of administration of drugs [6].

In a second most populous developing country like India there is a large unmet need for contraception as population control method. Most pregnancies result from non -use of contraception [4]. It is seen that instead of using regular, reliable contraceptive more women are choosing M.A., as post conception method. All thanks to convenience and ease of availability of M.A. drugs in addition to pharmaceutical advertizing of the same, more and more gynecologists are advocating safety of M.A. over surgical procedure.

A study conducted by Anke Hemmering, Freiderike Siedentopf \& Heribert Kentenich in German women, more women preferred Medical abortion over surgical because of positive outcome and high satisfaction levels, illustrating an improved accessibility of medical abortion [3]. It may be that the first solution to their conflict was the termination of pregnancy itself, and the convenience of it was the bonus. Less complications, easy accessibility of drugs at a reasonable cost and available as an outpatient procedure, medical abortion has rapidly become a popular method of getting rid of an unintended pregnancy. This has led to an abuse of drugs and a callous disregard to life, making more and more women use M.A as a way of handling unregulated fertility rather than use a reliable contraception.

Without doubt the demand for medical abortions is rising and most of these are not even done for the indications recommended by Government of India in the MTP act of 1972. Saseendran Pallikadavath and R. William Stones while studying social aspect of abortion in India whether it is due to need to produce male offspring that drives women to abortion found that, it is likely that unintended pregnancy, rather than the sex of the previous child, underlies demand for abortion in India. Rising educational attainment among women may lead to an increase in the demand for abortion [4]. In our experience we found that education level of a woman does matter in choosing abortion, for socio-economic, career and education reasons.

Another study, in Tamil Nadu, showed that nonconsensual sex, sexual violence and women's inability to refuse their husband's sexual demands were the factors that most frequently led to unwanted pregnancy and abortion [5]. We found that many women came forward for the termination of their pregnancies in an agitated state and demanded immediate resolution to their predicament, were pregnant as a result of non consensual sex. Many of the women had self administered the drugs and presented with its associated complications. We found that with easier access the guilt of abortion is reducing and it is being done for very trivial indications.

We are not averse to women's right to terminate their pregnancies, to let them abort the life which would change family dynamics, be a burden on family economics or be a reminder of an unpleasant event, but this has led to an alarming rise in demand of M A to the point of abuse.

\section{FIGURES AND TABLES}

Distribution within the sample - Demographic and Personal Preference

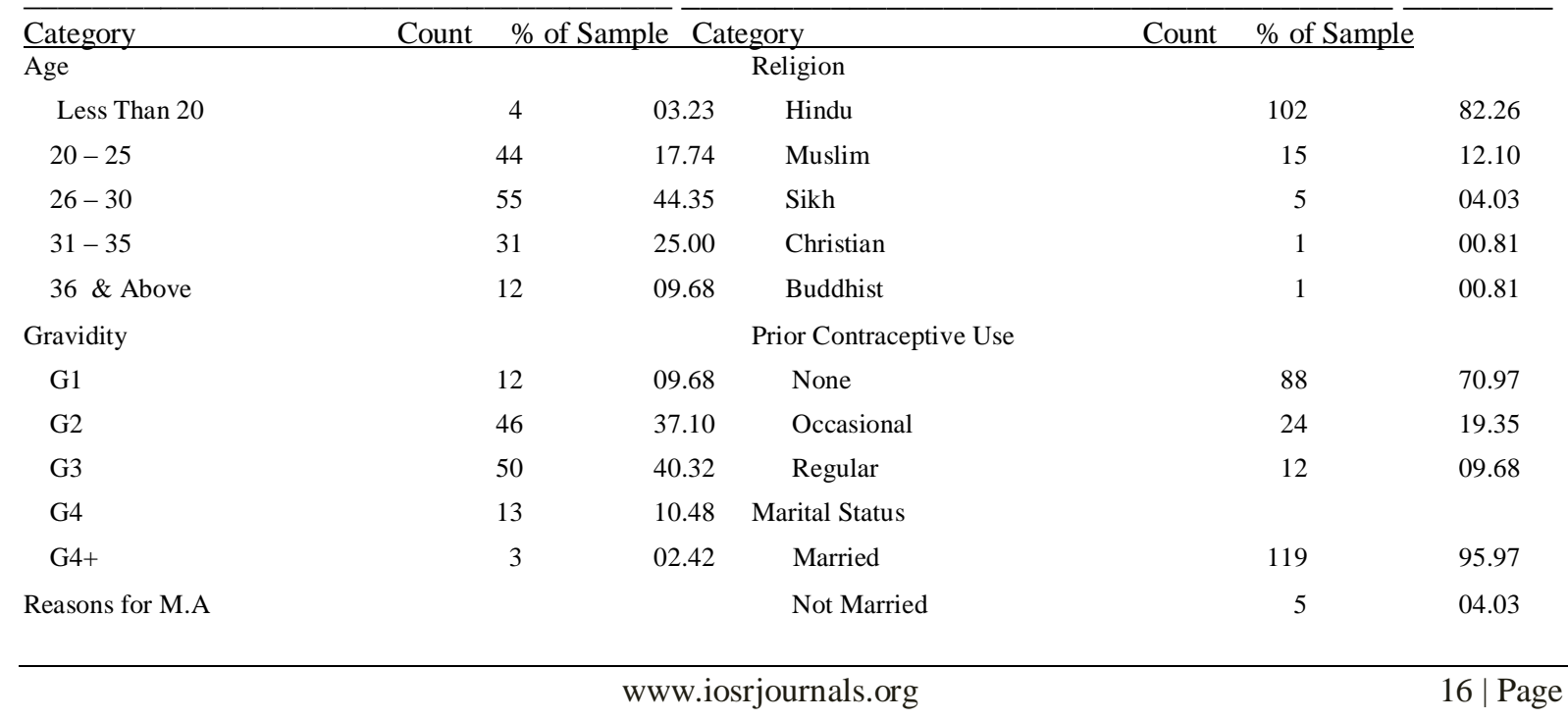


Too Soon Since LCB

Failed Contraception

Completed the Family

Economic / Career

Unmarried

$\begin{array}{ll}40.32 & \text { Willing to use Contraception } \\ 25.81 & \text { Yes } \\ 25.00 & \text { No } \\ 04.84 & \text { No Need } \\ 04.03 & \text { Maybe }\end{array}$

04.03
56.45

21.77

12.10

09.68

Table -1 Distribution by various factors among the Sample Population

Distribution within the sample - Psycho-Social Aspect

\begin{tabular}{lr}
\hline Category & \% of Sample \\
\hline Spouse / Family Support & \\
Yes & 89.00 \\
No & 11.00 \\
Mental Status & \\
Guilt & 06.00 \\
Anxiety & 10.00 \\
Fear & 18.00 \\
Neutral & 66.00 \\
Knowledge of M.A. & \\
Yes & 65.00 \\
No & 10.00 \\
Some & 25.00
\end{tabular}

Table -2 Distributions by Psycho-Social Aspects among the Sample Population

\section{VII.}

CONCLUSION

Medical abortion has become a safe and easy way of getting rid of unwanted pregnancies. This has led to wide spread misuse of drugs. What was intended as a safer alternative to surgical and criminal abortion is now available in the pharmacies, even without prescription.

The incidence of septic abortion may have reduced but self administration of drugs without proper protocol or dosage is leading to more complication like incomplete abortion, profuse vaginal bleeding and its attendant morbidity. Since it is easy and convenient the attitude towards the unintended pregnancies are changing. The moral obligation to life, guilt and anxiety is reducing. The need for responsible contraception is not there anymore in urban women, because they have easily available, cheap, method of abortion with low complications.

Whether we want it or not M.A is here to stay! And as long it is freely available women will not be serious about contraception! Women should have the freedom to choose abortion, if the birth of an unintended child is going to change family dynamics and economy. But should they be allowed to have as many M.A as they can have? Should gynecologists have a say in the matter to influence their minds regarding, continuation of pregnancy or abortion, the use of pre or post abortion contraception? Do they have right to insist up on permanent method of contraception, like sterilization upon completion of family? These are difficult and moral questions that need to be answered - after all it is the choice of the couple, not that of the provider.

\section{REFERENCES}

[1]. N .Sheriar, Accessing safe abortion, Journal of Family Welfare ,vol 50,special issue,2007

[2]. Etul Bataya,Sheriar Nozer,Anand Abhijeet, Philip Neena,Are obstetrician and gynecologists awarwe of Medical abortion in India?Journal of Obstetrics and Gynecology Of India, vol.,56,no.4,August 2006,349-345

[3]. Anke Hemmerling, Freiederike Siedentopf, \&Heribert Kentenich Emotional impact and acceptability of medical abortion with mifepristone: A German experience, Journal of Psychosomatic Obstetrics \& Gynecology, March 2005; 26(1): 23-31ISSN 0167-482X print/ISSN 1743-8942 online \# 2005 Taylor \& Francis Group Ltd

[4]. Saseendran Pallikadavath and R. William Stones ,Maternal and Social Factors Associated with Abortion in India: A PopulationBased Study, International Family Planning Perspectives Volume 32, Number 3, September 2006

[5]. Ravindran TK and Balasubramanian P, "Yes" to abortion but "no" to sexual rights: the paradoxical reality of married women in rural Tamil Nadu, India, Reproductive Health Matters, 2004, 12(23):88-99.

[6]. Ulmann, A., Silvestre, L., Chemama, L., Rezvani, Y., Renault, M., Aguillaume, C. J. and Baulieu, E.-E. (1992), Medical termination of early pregnancy with mifepristone (RU 486) followed by a prostaglandin analogue: Study in 16, 369 women. Acta Obstetricia et Gynecologica Scandinavica, 71: 278-283. doi: 10.3109/00016349209021052, Available at website http://www.ncbi.nlm.nih.gov/pubmed/1322621 